\title{
Discrimination and generalization of leaf damage by blue jays (Cyanocitta cristata)
}

\author{
PAMELA G. REAL, RUTH IANNAZZI, and ALAN C. KAMIL \\ University of Massachusetts, Amherst, Massachusetts \\ and \\ BERND HEINRICH \\ University of Vermont, Burlington, Vermont
}

\begin{abstract}
Blue jays (Cyanocitta cristata) responded to projected black-and-white silhouettes of cherry leaves that were either undamaged or were damaged by either cryptic caterpillars that disguise leaf damage due to their feeding or by noncryptic caterpillars that do not disguise leaf damage due to their feeding. Pecks to the key on which the images were projected were reinforced only if interresponse times fell within specified temporal boundaries. These boundaries were different in the presence of the two types of leaf damage. Following training with one exemplar of each damage type, the jays correctly categorized novel instances of both types. They categorized novel undamaged leaves as if they were examples of leaves showing damage by cryptic caterpillars. Results suggest that the jays can readily discriminate and generalize to new members of a polymorphous stimulus class. The results suggest that techniques for the study of concept formation in animals can be applied to an animal's ability to categorize stimuli that are involved in a potential prey-predator relationship.
\end{abstract}

Many palatable, highly cryptic, leaf-eating caterpillars have feeding behaviors that appear to disguise or eliminate evidence of leaf damage due to feeding. These caterpillars pare leaves evenly, often snipping off the leaves on which they have fed. Unpalatable, noncryptic caterpillars lack these behaviors. These caterpillars leave abundant evidence of their feeding. The leaves on which unpalatable caterpillars have fed are often left in tatters (Heinrich, 1979). Heinrich and Collins (1983) have suggested that the feeding stategies of the cryptic caterpillars may have evolved under predation pressures to further reduce their visibility, and they have demonstrated that black-capped chickadees (Parus atricapillus) can use both artificial and naturally occurring leaf damage as a means of localizing prey items. Their data indicate that birds are capable of discriminating leaf damage and that leaf damage may determine the locus of foraging efforts. Their data suggest, more indirectly, that foraging behaviors may be determined, at least in part, by leaf or leaf-damage characteristics as a result of

This research was supported, in part, by NSF Grant BNS8102335 to the University of Massachusetts and by NSF Grant DEB-8116662 to the University of Vermont. We would like to thank Betsy Frederick and Anne Quackenbush for their assistance in running animals. Thanks are due Joe Ayres, Ernie Dzendolet, Dave Palmer, and Deb Olson for helpful discussions and comments and R. J. Herrnstein for several points of clarification. Reprints may be obtained from P. G. Real, Department of Psychology, Middlesex House, University of Massachusetts, Amherst, MA 01003. differential association of different types of leaves or leaf damage with the presence or absence of palatable prey items.

The diversity of leaf geometry within species of tree, the similarity between species of trees, the diversity introduced by seasonal variations in prey availability, and the differences in leaf geometry due to the feeding activity of caterpillars pose a potentially formidable discrimination task for birds. The data reported by Heinrich and Collins (1983) further suggest, in the language of the psychology of learning, that birds may be able to behave differentially in the presence of instances of one or more polymorphous stimulus classes and to generalize correctly in the presence of new instances. Polymorphism, here, refers to stimuli not easily quantifiable on the basis of a single physical continuum or easily specifiable by a single attribute that is sufficient or necessary for inclusion of a stimulus item in a given class (see Herrnstein, 1982, 1984, for a detailed discussion of this issue).

The adaptive significance of an organism's ability to categorize biologically relevent stimuli correctly and to form categories that are sufficiently broad to encompass naturally occurring variation, yet sufficiently narrow to prohibit false inclusions has been discussed by Marler (1982) and others (Herrnstein, 1979, 1982; Herrnstein, Loveland, \& Cable, 1976; Staddon, 1975). It has been demonstrated that pigeons can easily categorize projected color-slide images containing people (Herrnstein, 1979), trees 
(Herrnstein et al., 1976), water (Herrnstein, 1979), fish (Herrnstein \& de Villiers, 1980), and letters of the alphabet (Blough, 1984; Morgan, Fitch, Holman, $\&$ Lea, 1976), but not food cups, wheeled vehicles (Herrnstein, unpublished data), or line drawings of Charlie Brown (Cerella, 1980), to cite a few of the successes and failures. Although the adaptive significance of the ability to form categories may be independent of the specifics of a stimulus class, the biological relevance of the stimuli used in previous studies is uncertain.

The present experiment employed a modification of Herrnstein's technique for the study of concept formation in an effort to investigate a potential preypredator relationship. Our concern was not with the limits of what can define a perceptual category. Rather, we addressed the specific proposal suggested by Heinrich and Collins (1983). That is, can blue jays (Cyanocitta cristata) discriminate the two types of leaf damage? Can they generalize from a single exemplar of each type to novel instances that capture some of the naturally occurring variation that is to be found? Can leaves damaged by cryptic palatable caterpillars appear, to the jays, to be more like undamaged leaves as a result of the feeding strategies of these caterpillars? And is it reasonable to assume that the leaves on which these cryptic caterpillars feed would be harder to differentiate from undamaged leaves, on the basis of visual cues, by a potential predator in nature?

\section{METHOD}

\section{Subjects}

Four adult blue jays (Cyanocitta cristata) approximately 1 year old at the start of the experiment, served as subjects. They had been taken from their nests at 10-12 days of age and hand-reared in the laboratory. All birds were experimentally naive at the start of the experiment and were maintained at $\mathbf{8 0 \%}$ of their adult freefeeding weights throughout the experiment.

\section{Apparatus}

A homemade pecking panel, mounted in a sound-attenuated chamber (BRS-LVE), provided an experimental enclosure $36.5 \mathrm{~cm}$ high, $35 \mathrm{~cm}$ wide, and $30.5 \mathrm{~cm}$ deep. The opening of the food magazine was $13 \mathrm{~cm}$ from the floor of the chamber, $3.5 \mathrm{~cm}$ high, $4.5 \mathrm{~cm}$ wide, and located in the center of the front panel. A 7.5-cm-high $\times 11.6-\mathrm{cm}$-wide rectangular pecking key was mounted $13.5 \mathrm{~cm}$ from the floor and $4 \mathrm{~cm}$ from the left wall of the chamber. The panel also contained a small round pecking key that was not used in the present experiment. The panel was equipped with a houselight in the upper left-hand corner, and a perch was located $10 \mathrm{~cm}$ from the front panel and $4 \mathrm{~cm}$ from the floor. Masking noise was provided through a speaker located behind the panel and by a ventilating fan at the rear of the chamber.

Experimental stimuli were rear-projected onto the rectangular pecking key by a Kodak Carousel projector $(650 \mathrm{H})$. Reinforcement, in the form of one-half of a mealworm, was provided by a Davis Universal feeder. Experimental events and recording were arranged by a Data General Nova computer and standard 28-V dc electromechanical equipment.

\section{Preparation of Stimuli}

Experimental stimuli were slides of black-and-white silhouettes of choke cherry leaves (Prunus virginiana) exhibiting leaf damage due to feeding by the cryptic, palatable small-eyed sphinx (Paonio myops) ("neat") or by the noncryptic, unpalatable tussock moth caterpillar (Hemerocampa leucostigma) ("messy") (see Heinrich, 1979), or leaves showing no detectable damage due to predation, breakage, etc. The leaves were traced on black construction paper from colored slides taken in the field of leaves on or adjacent to which either palatable or unpalatable caterpillars were feeding. The paper leaves were placed on a white background and photographed with a Nikkor-H camera equipped with a $50-\mathrm{mm}$ lens on 35-mm Kodalith Ortho Film 6556, Type 3 (Kodak), a high-contrast black-and-white negative slide film, and then reproduced as black-on-white positives. Each leaf was photographed in rotations along vertical and horizontal axes (with the stem pointing north, south, east, and west) and as mirror images of the same rotations. Stimuli were projected onto the rectangular key through a 0.90 Wratten neutral density filter.

\section{Procedure}

Preliminary training. The jays were hand-shaped to peck the rectangular key (slide key) in the presence of either a red or a green slide which, when rear-projected, covered the key completely. Following keypeck training, each peck at the slide key was reinforced for three sessions. Each peck extinguished the slide key, illuminated the feeder light for $3 \mathrm{sec}$, delivered one-half of a mealworm, and initiated a 2.5-sec delay during which the houselight remained illuminated and the projector advanced. Daily experimental sessions, conducted 6 days per week, consisted of 30 randomized presentations of red and green slides, the colors occurring with equal frequency. Pecks to the slide key during feeder light operation or during the delay had no scheduled consequences.

When pecking to both colors occurred reliably, a discrete-trials response pacing contingency was imposed. That is, keypecks were reinforced only when the time between successive responses (interresponse times, IRTs) fell within specific temporal boundaries. The difference between the upper bound of the IRTs required in the presence of red and the lower bound of the IRTs required in the presence of green was gradually increased over the course of approximately 30 experimental sessions. The number of correct IRTs (fixed ratio, FR) required to produce reinforcement was varied from one to three. When the FR requirement was greater than one, feedback was not given for successive correct IRTs. Maximum trial duration was $5 \mathrm{~min}$. If subjects failed to produce the required number of criterion IRTs within that time, the trial ended without reinforcement. When criterion was met, reinforcement terminated the trial. IRTs were timed from each response that occurred after the first $5 \mathrm{sec}$ of each trial, and responses during the first $5 \mathrm{sec}$ were recorded but had no scheduled consequences. The final response requirements at this stage of training were as follows: in red, $2 \mathrm{sec}>$ IRT $<4 \mathrm{sec}$; in green, $5 \mathrm{sec}>$ IRT $<20 \mathrm{sec} ; \mathrm{FR}=3$.

Discrimination training. The jays were then trained on the same response pacing contingency in the presence of a single example of each of the two damage types ("neat," damage created by cryptic, palatable caterpillars, and "messy," damage created by noncryptic, unpalatable caterpillars). Sessions consisted of 40 trials, and each leaf was shown 20 times, with all rotations represented approximately equally often. The training leaves are shown in Figure 1 (above the " $T$," in the two rows labeled "neat" and "messy"). Contingencies were in all respects as they were during red and green; however, the IRT contingency was relaxed initially to accommodate some lack of responding due to the novelty of the stimuli, and then increased rapidly. FR requirement was varied from one to three, as in the previous phase. At the end of this stage of training, the IRT contingency was as follows: in the presence of "messy," $2 \mathrm{sec}>$ IRT $<4 \mathrm{sec}$; in the presence of "neat," $6 \mathrm{sec}>$ IRT $\leqslant 12 \mathrm{sec}$. Maximum trial time was $40 \mathrm{sec} ; \mathrm{FR}=1$. 


\section{war 10116010 "MESSY" 4 93 5 14 6 \\ PROBE

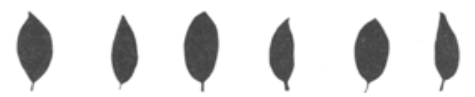

Figure 1. Experimental stimuli for all stages of the experiment. Leaves used during training and during generalization are shown in the top two rows. The row labeled "neat" shows the leaves with damage due to feeding by cryptic, palatable caterpillars. The row labeled "messy" shows the leaves with damage due to noncryptic, unpalatable caterpillars. Training leaves are marked "T," generalization leaves are A-F, and ambiguous leaves are marked "G." Undamaged probes are on the bottom row, and are displayed in order of their presentation (from left to right).

When all subjects showed reliable and clear differentiation of IRTs in the presence of the two stimulus types, trial time was decreased gradually and the time between the lower and upper bounds of the two IRT classes was reduced until all subjects were unable to meet a criterion of a single correct IRT on no fewer than $10 \%$ but no more than $25 \%$ of the 40 daily trials. The final schedule parameters were as follows: For jays 60, 61, and 62 , maximum trial time was $20 \mathrm{sec}$, and, in the presence of "messy" leaves, $2.3 \mathrm{sec}>$ IRT $<2.6 \mathrm{sec}$ was reinforced and, in the presence of "neat" leaves, $6 \mathrm{sec}>$ IRT $<8 \mathrm{sec}$ was reinforced; for jay 63, the procedure was identical except that maximum trial time was $22 \mathrm{sec}$ and, in the presence of "messy" leaves, $2.4 \mathrm{sec}>$ IRT < $2.6 \mathrm{sec}$ was reinforced. For all jays, $F R=1$. During this period, subjects were shown one of four randomized sequences of slides, which were recycled so that subjects saw the same sequence approximately every fifth session except where noted (novel sequences were introduced occasionally to assess the possibility that the subjects had learned particular slide sequences.)

Generalization testing. When the distribution of IRTs in the presence of the training slides had stabilized, as judged by visual inspection, the subjects were exposed to 5 consecutive days of generalization testing. During generalization testing, novel leaves showing each of the damage types were substituted for some of the training stimuli (four of each type). Novel stimuli consisted of a single leaf of each type in four, north, south, east, and west, rotations. That is, eight slides were substituted each day, four of each damage type, but only one leaf of each type. On the 5th day of generalization testing, an entirely novel sequence of the training stimuli was presented with the substituted eight generalization stimuli. Leaves used during generalization testing are shown in Figure 1 in the rows labeled "neat" and "messy." The letters A-F indicate the pairs that were substituted. The subjects viewed the stimuli, on successive testing days, in the following order: jay 63, ABCDE; jays 61 and 62, BCDEA; and jay 60, BCDEF. Jay 60 initially viewed set $A$ and then began experiencing difficulty with the IRT contingency. This subject was run an additional five sessions on the baseline discrimination training procedure before viewing B-F. Data from exposure to set A, for this subject, are not included in the analyses that follow. During this phase of the experiment, reinforcement was available for IRTs that fell within the bounds specified for a given leaf damage type on both trial types (old and novel, see Discrimination training).

Following probe trials (see below) and five sessions on baseline training slides, the jays were given one session of a novel sequence of slides with one novel leaf of each type (set G, Figure 1) in two compass angle rotations (four slides) substituted for four training slides, two of each type. These leaves were chosen so as to be as similar as possible to one another, and as dissimilar as possible to the training slides. Prior to this session, generalization test stimuli were chosen without regard to their similarity to training stimuli or to each other.

Probe trials. Following generalization testing, all subjects were returned to the original discrimination training slides for five consecutive sessions. The subjects were then exposed to sessions during which a single undamaged leaf, in four rotations, was substituted for some of the training stimuli (two of each type). In contrast to the procedure that was followed during generalization tests, IRTs in the presence of the novel undamaged leaves were recorded but were never reinforced. The subjects were exposed to two consecutive sessions of probe trials, followed by one session of baseline training slides and then by two sessions of probe trials, one session of baseline, and two sessions of probe trials. Thus, six undamaged leaves were viewed on a total of 24 probe trials. Reinforcement continued to be available for correct IRTs in the presence of the training stimuli. The stimuli used in probe trials are shown in Figure 1.

\section{RESULTS}

Figure 2 shows the mean relative frequency of IRTs of different durations in the presence of "messy" leaves for all four birds. Individual subjects are shown in columns. Data are from the original discrimination procedure with the two training leaves and the 5 days of generalization testing. Note that the scale on the $\mathrm{x}$ axis is arranged, for each bird, so that criterion IRTs are displayed in a single class interval, marked by a vertical dashed line. Daily relative frequencies were averaged in the top row (original discrimination training) and the middle row (training stimuli on generalization days). Relative frequencies were computed from grand totals of IRTs summed across days in the bottom row [all generalization trials (filled circles) and first viewing of all generalization stimuli (unfilled circles)]. The distribution of IRTs in the presence of the "messy" training stimulus showed good conformity to pacing contingencies. Modal IRTs fell within one class interval $(0.2-0.3 \mathrm{sec})$ short of the lower bound of the criterion class interval in all cases. With the exception of jay 63 , the birds showed some bursting; that is, IRTs shorter than $0.2 \mathrm{sec}$. Performances during generalization testing, on both novel and old stimuli and during baseline training were virtually identical in every respect. Data from the first exposure to the novel stimuli within an experimental session captured the essential features of performance averaged over all generalization trials. As mentioned in the procedure, the response pacing contingency was sufficiently difficult for not all trials to terminate in reinforcement; however, there is no obvious systematic relationship between the novelty of a stimulus on the first exposure and the relative frequency of IRTs in the criterion class interval.

Figure 3 shows the mean relative frequency of IRTs of different durations in the presence of "neat" 

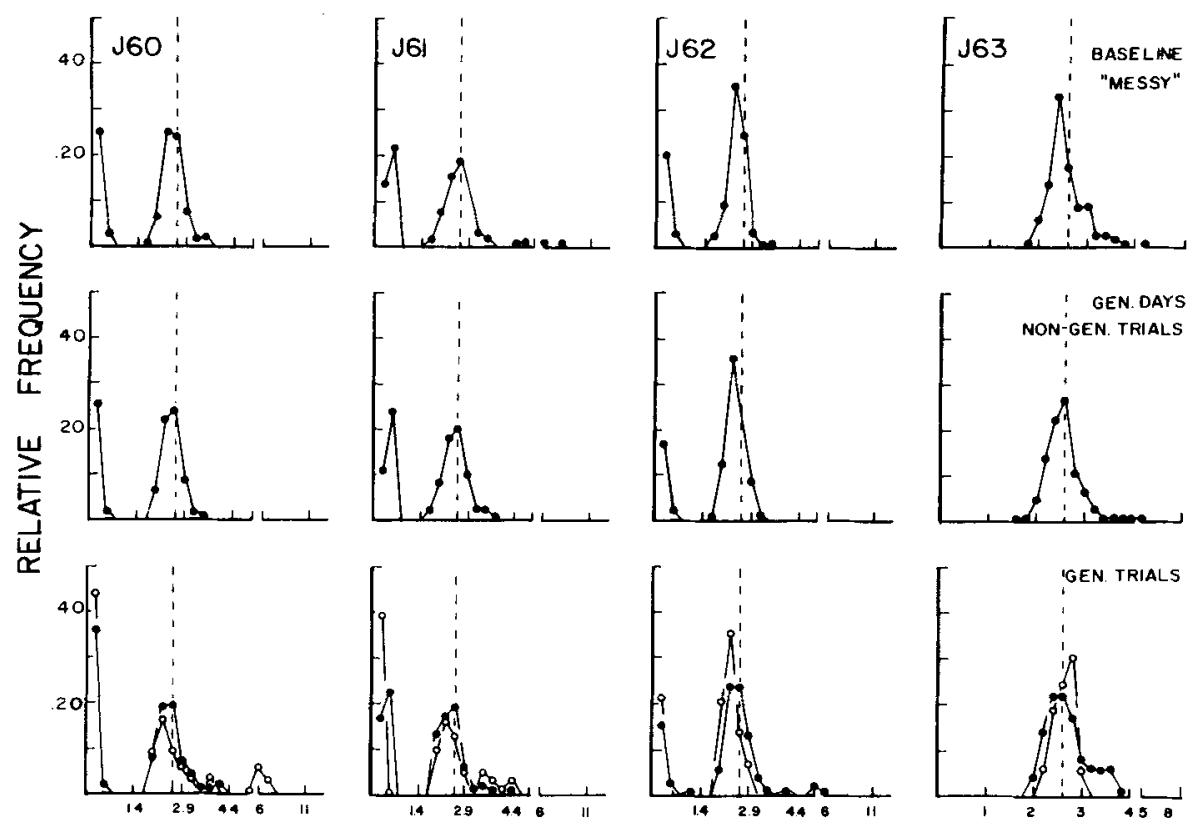

CLASS INTERVAL (sec)

Figure 2. Relative frequency of IRTs in the presence of "messy"' slides. Class intervals are marked on the $x$-axis. Iindividual subjects are shown in columns. The first class interval shows IRTs 0.0-0.2 sec. Thereafter, for jays 60-62, class intervals are in 0.3-sec increments; for jay 63, class intervals are in 0.2-sec increments. The scale on the x-axis is arranged, for each bird, so that criterion IRTs are displayed in a single class interval. Following the break in the $x$-axis, class intervals are in 1-sec increments. The top row shows mean relative frequency from the last 3 days of baseline prior to generalization; the middle row shows mean relative frequency from generalization days on trials with training stimuli; and the bottom row shows mean relative frequency on all generalization trials (filled circles) and on first viewing of all generalization stimuli (unfilled circles).
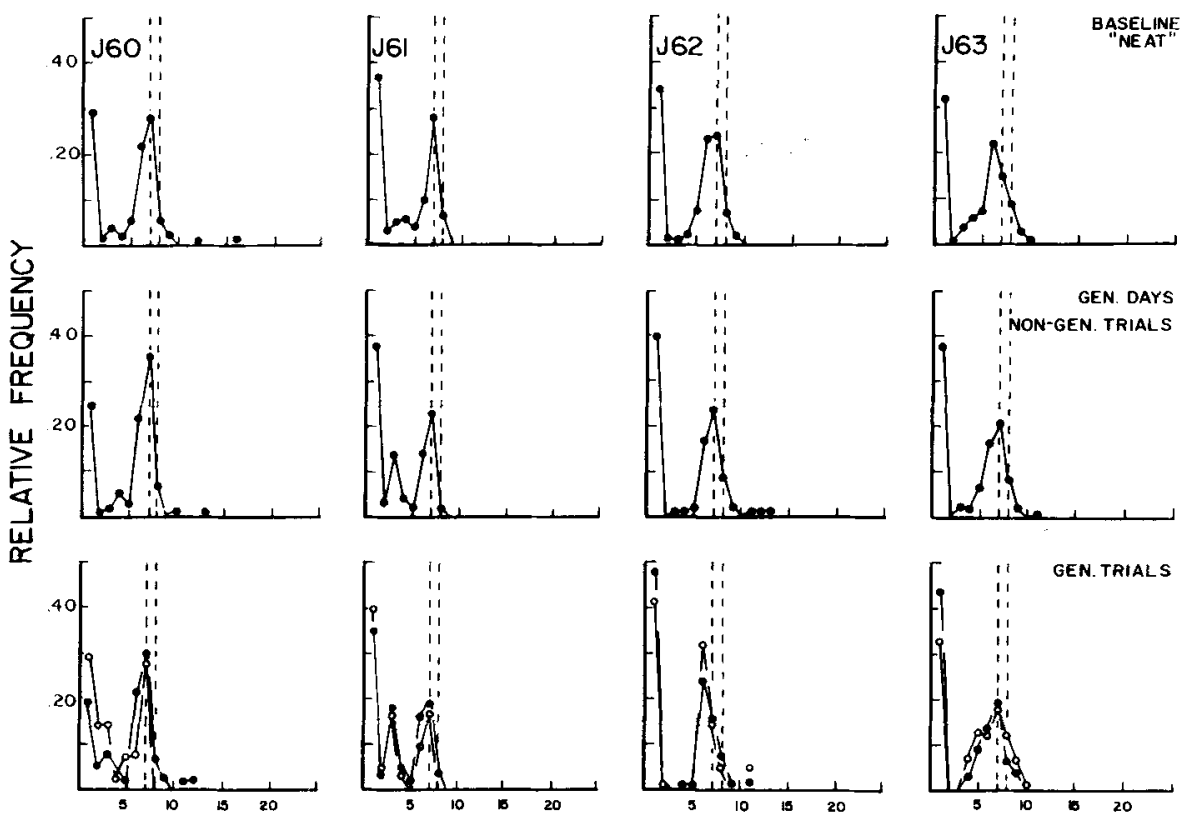

CLASS INTERVAL (sec)

Figure 3. Relative frequency of IRTs in the presence of "neat" slides. Class intervals are in 1-sec increments. Details are as in Figure 2 except that criterion IRTs are displayed with upper and lower bounds indicated by the vertical dashed lines. 
leaves for all four birds. Rows are the same as in Figure 2. All class intervals are in 1-sec increments. Criterion class intervals are marked by the vertical dashed lines and are inclusive. All birds showed good conformity to the response pacing contingencies in presence of "neat" leaves. All birds showed more bursting on trials with "neat" leaves than on trials with "messy" leaves. A comparison of Figures 2 and 3 reveals good differentiation of IRTs in the presence of the two damage types. Jay 61 , however, showed some lack of differential responding on "neat" trials. A relatively high frequency of $2-3$-sec IRTs was evident in this bird's performance. As on trials with "messy"' leaves, performance during generalization testing overall and on first viewing of each novel "neat" leaf were highly similar, suggesting that performance on generalization trials was not primarily the result of learning due to reinforcement for correct IRTs on trials with novel stimuli.

Figure 4 shows the relative frequency of IRTs during the return to the baseline discrimination procedure after generalization testing (top row, "messy" training trials; middle row, "neat" training trials) and on probe trials with undamaged leaves (bottom row). Note that in the top row, consecutive class intervals are the same as those in Figure 2 (training with "messy" leaves), and 'that in the middle row, consecutive class intervals are the same as those in Figure 3 (training with "neat" leaves). In the bottom row, class intervals are the same as in the middle row in order to facilitate a comparison of performances on baseline "neat" trials and on trials with undamaged probes. The resolution in the data in the top row is much greater, despite the apparent similarity of the rows. Criterion class intervals on trials with training stimuli are shown by the vertical dashed lines. It is clear that performance during the return to baseline discrimination (filled circles) and in the presence of training stimuli on probe days (unfilled circles) was unchanged from original baseline performance for all birds. Furthermore, performance on probe trials was virtually identical to performance on "neat" trials. Jay 61 continued to show a high frequency of IRTs appropriate for "messy" trials in the presence of undamaged leaves. Only one subject (jay 63) showed an increase in the variability of IRTs on probe trials. This subject showed an increase in the frequency of IRTs shorter than $6 \mathrm{sec}$ and longer than $8 \mathrm{sec}$.

Figure 5 shows IRTs from the four novel trials in the session with ambiguous stimuli (set G, Figure 1). Each trial type is marked on the $\mathrm{X}$-axis, $\mathbf{N}$ indicates "neat," $M$ indicates "messy," and the subscripts
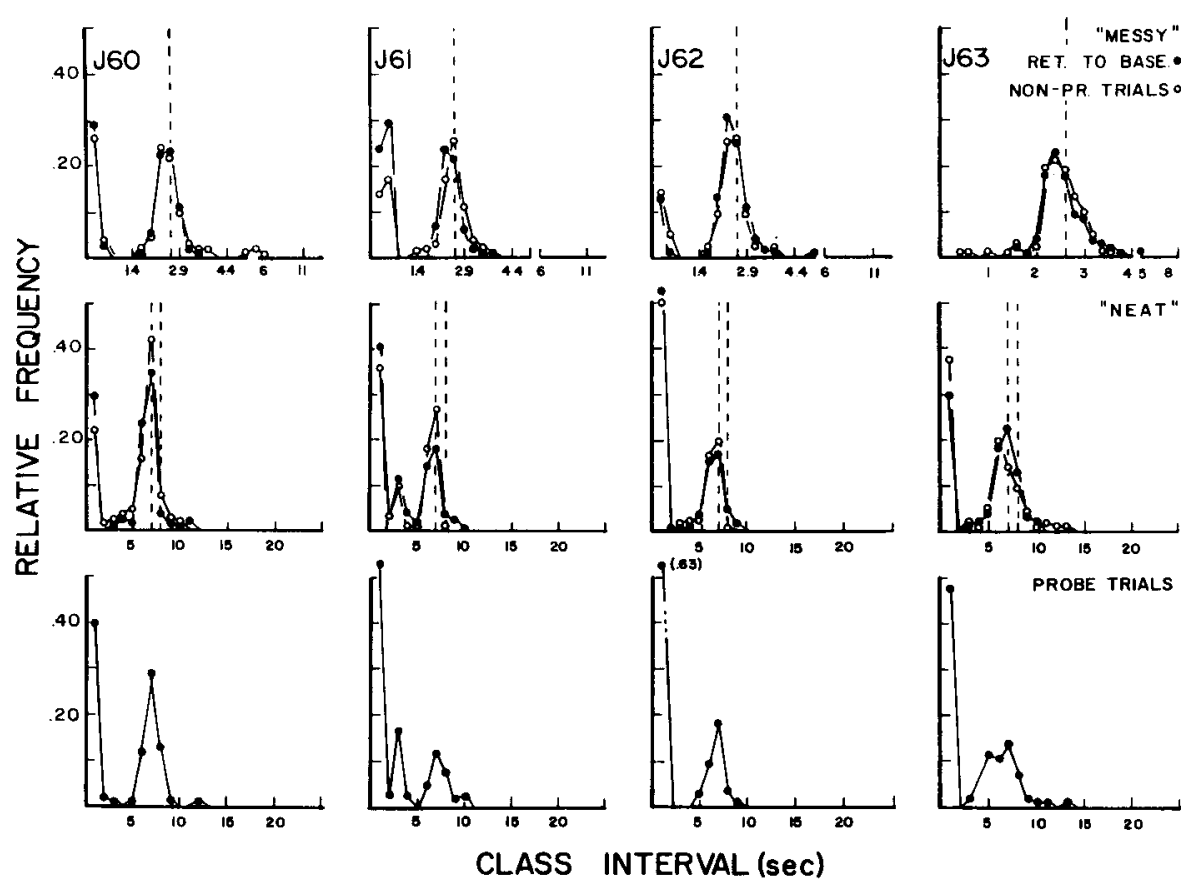

Figure 4. Relative frequency of IRTs during the return to baseline discrimination after generalization and during sessions with probe trials with undamaged leaves. The top row shows performance on "messy" slides; the middle row shows performance on "neat" slides. The mean relative frequency from the 5 days of the return to baseline procedure after generalization is shown by filled circles. Training-stimulus trials on probe days are shown by the unfilled circles. The bottom row shows performance on probe trials. Class intervals on the top row are the same as in Figure 2 ("messy") and are shown in much greater resolution than the bottom two rows. The class intervals on the bottom two rows are the same as in Figure 3 ("neat"). 


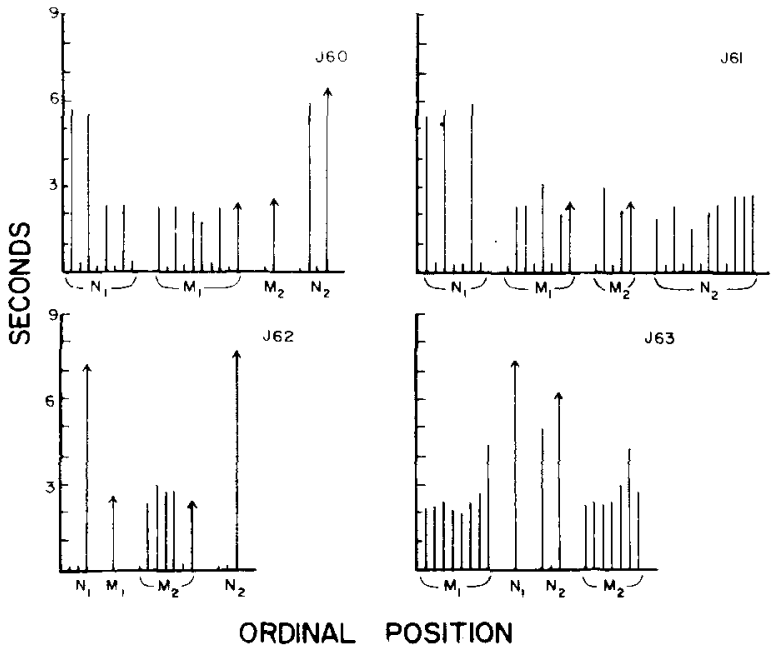

Figure 5. IRTs in consecutive ordinal position from trials with ambiguous stimuli (set $\mathbf{G}$, Figure 1). Trial type is marked $\mathbf{N}$ or $\mathbf{M}$, and subscripts indicate the order in which the four novel slides were viewed. Arrows indicate reinforced IRTs.

indicate the order in which the four novel slides were interspersed in the sequence of training slides. Arrows indicate IRTs that were reinforced, thus terminating the trial. All IRTs are shown in ordinal position from the beginning to the end of the trial. Subject numbers are shown in the upper right-hand corner.

All subjects correctly categorized these stimuli according to type on first viewing. Jay 60 correctly categorized the first "neat" leaf viewed with its initial IRTs and then shifted to IRTs appropriate to "messy" leaves. This subject then correctly categorized the remaining three novel stimuli. Jay 61 showed obvious incorrect categorization on second viewing of the "neat" leaf after correctly categorizing the leaf on first viewing but failing to meet the criterion for reinforcement. The other two subjects correctly categorized the leaves on all novel trials, whether or not criteria for reinforcement were met on previous novel trials. Thus, the behavior of only one subject (jay 61) suggests the possibility of withinsession learning due to failure or success in meeting the criteria for reinforcement on trials with novel stimuli.

\section{DISCUSSION}

The present experiment was designed to assess whether blue jays were able to discriminate and generalize leaf damage. The procedure involved training the jays to pace their responses according to leafdamage type in the presence of a single black-andwhite silhouette of a leaf showing damage due to feeding by a cryptic, palatable caterpillar and a single silhouette of a leaf showing damage due to feeding by a noncryptic, unpalatable caterpillar. The jays were then exposed to a series of tests with novel instances of both damage types or novel instances of undamaged leaves. The jays informed us of their categorization of each novel stimulus by their choice of one of the two previously reinforced response patterns. The results indicate that the jays were capable of correctly categorizing novel instances of each damage type in spite of the fact that the novel slides showed substantial variation from original training stimuli and that they did so prior to receipt of reinforcement for responding to the novel stimuli. For three of the four jays in this study, this result is completely unambiguous. For the fourth jay (jay 61), there was some lack of differentiation of IRTs in the presence of "neat" leaves. None of the birds, however, showed substantial departures from baseline performance in the presence of novel stimuli showing the two damage types. Furthermore, all jays responded to novel stimuli showing no leaf damage as they had previously responded to leaves that were damaged by cryptic, palatable caterpillars. The results of the study are in general agreement with Heinrich and Collins's (1983) demonstration that birds can use leaf-damage as a means of localizing prey and are consistent with the hypothesis that cryptic caterpillars provide fewer visual cues to distinguish the leaves on which they feed from undamaged leaves. Heinrich and Collins (1983) have proposed that the feeding strategies of these cryptic caterpillars may have evolved under specific predation pressure from birds, for whom vision is a dominant sensory modality.

It is clear that none of the birds in the present study showed substantial deviation from the two reinforced response patterns on trials with novel stimuli. This result is dissimilar to previous research on generalization along easily quantifiable physical continua (i.e., wavelength, brightness) showing continuous generalization gradients and dissimilar to previous concept formation work with pigeons (Herrnstein, 1979) showing rank-ordering of complex visual stimuli following training with variableinterval (VI) reinforcement of positives and nonreinforcement of negatives. Although the differences may be due to the nature of the stimuli used in the present experiment-silhouettes-Cerella (1979) obtained rank-ordering to silhouettes of oak leaves using VI reinforcement of positives. It is more likely that the differences are due to the response pacing contingency in the present experiment. Data reported by Blough (1963) and others (Crowley, 1979; Cumming \& Eckerman, 1965; Migler, 1964) suggest that continuous generalization gradients may be due to the mixing of discrete patterns of responding with other behaviors to produce intermediate rates of responding. It has been hypothesized that when a temporal property of a response (i.e., IRT, duration) is reinforced differentially in the presence of two 
stimuli that vary along one physical dimension, intermediate durations do not occur to intermediate stimuli (see Crowley, 1979), although intermediate response rates may occur (see Herrnstein \& van Sommers, 1962). Although it appears that the birds in the present study were "locked" into the two reinforced response patterns, this result cannot be taken as an explanation of their correct categorization of novel stimuli, since they were free to make errors.

It is also clear, from examining the test stimuli, that there is no simple rule of geometry that could lead to correct categorization of all of the novel stimuli. Size, or general shape of a leaf that is better preserved in the "messy" leaves, for example, could have been used to differentiate "neat" from "messy" on generalization trials, but would have produced incorrect categorization of about half of the undamaged probes. Locus, or detail, of damage was highly variable in both damage types. The presence or absence of "holes" or "jagged edges" could differentiate "neat" from "messy," yet the feature that distinguishes "messy" leaf A (Figure 1, the hole in the middle) from "neat," for example, is not represented in the training stimulus. From the standpoint of the literature on concept formation or categorization in animals, these data represent another example of spontaneous, rather than inductive, categorization, since the initial discrimination training with a single exemplar of each of the damage types need not have represented a sufficient condition for the correct generalization from the training stimuli to the novel ones. It has been suggested (Cerella, 1979; Herrnstein, 1982, 1984) that the spontaneous categorization of stimuli showing a range of naturally occurring variation may represent one aspect of the biological adaptation of an organism to its environment. Further, it has been suggested that these categories may be divided along "taxonomic" lines (Cerella, 1979, p. 76) or at the "species or generic level" (Herrnstein, 1982, p. 115). We offer the suggestion that the appearance of generic categorization may be due, in part, to the choice of stimuli in previous experiments (i.e., trees, fish, people, water). We know of no published accounts of an organism's ability to categorize naturalistic stimuli that vary at the level of detail used in the present experiment. Yet, it is clear that the blue jays experienced little or no difficulty with this task, regardless of the specifics of how the categories were formed.

\section{REFERENCES}

Blough, D. S. (1963). Interresponse time as a function of continuous variables: A new method and some data. Journal of the Experimental Analysis of Behavior, 6, 237-246.

Blough, D. S. (1984). Form recognition. In H. S. Terrace, T. G. Bever, \& H. L. Roitblat (Eds.), Animal cognition. Hillsdale, NJ: Erlbaum.

Cerella, J. (1979). Visual classes and natural categories in the pigeon. Journal of Experimental Psychology: Human Perception and Performance, 5, 68-77.

Cerella, J. (1980). The pigeon's analysis of pictures. Pattern Recognition, 12, 1-6.

Crowley, M. A. (1979). The allocation of time to temporally defined behaviors: Responding during stimulus generalization. Journal of the Experimental Analysis of Behavior, 32, 191-197.

Cumming, W. W., \& Eckerman, D. A. (1965). Stimulus control of a differentiated operant. Psychonomic Science, 3, 313-314.

Heinrich, B. (1979). Foraging strategies of caterpillars: Leaf damage and possible predator avoidance strategies. Oecologia, 42, 325-337.

Heinarch, B., \& Collins, S. L. (1983). Caterpillar leaf damage, and the game of hide-and-seek with birds. Ecology, 64, 592-602.

Herrnstein, R. J. (1979). Acquisition, generalization and discrimination reversal of a natural concept. Journal of Experimental Psychology: Animal Behavior Processes, 5, 116-129.

Herrnstein, R. J. (1982). Stimuli and the texture of experience. Neuroscience and Biobehavioral Reviews, 6, 105-117.

Herrnstein, R. J. (1984). Object, categories, and conditioned stimuli. In H. S. Terrace, T. G. Bever, \& H. L. Roitblat (Eds.), Animal cognition. Hillsdale, NJ: Erlbaum.

Herrnstein, R. J., \& de Villiers, P. J. (1980). Fish as a natural category for people and pigeons. In G. H. Bower (Ed.), The psychology of learning and motivation (Vol. 14). New York: Academic Press.

Herrenste in, R. J., Loveland, D. H., \& Cable, C. (1976). Natural concepts in pigeons. Journal of Experimental Psychology: Animal Behavior Processes, 2, 285-302.

Herrnstein, R. J., \& van Sommers, P. (1962). A method for sensory scaling with animals. Science, 135, 40-41.

Marler, P. R. (1982). Avian and primate communication: The problem of natural categories. Neuroscience and Biobehavioral Reviews, 6, 87-94.

Migler, B. (1964). Effects of averaging data during stimulus generalization. Journal of the Experimental Analysis of Behavior, 7, 303-307.

Morgan, M. J., Fitch, M. D., Holman, J. G., \& Lea, S. E. G. (1976). Pigeons learn the concept of an 'A.' Perception, 5, 57-66.

Staddon, J. E. R. (1975). A note on the evolutionary significance of "supernormal" stimuli. The American Naturalist, 109, 541-545.

(Manuscript received October 28, 1983; revision accepted for publication March 5, 1984.) 